Geusaer Str., 06217 Merseburg

Tel.: +49 (0) 3461 / 462907

Fax: +49 (0) $3461 / 462919$

E-Mail: renate.patz@ltg.fh-merseburg.de

\section{Nachhaltige Entwicklung und Innovation im Energiebereich}

\author{
von Gerd Hanekamp, Europäische Akade- \\ mie Bad Neuenahr-Ahrweiler
}

Innovationen gelten in vielen Bereichen als Vorbedingung für eine nachhaltige Entwicklung. Sie sollen einen steigenden Bedarf mit einer Reduktion des Ressourcenverbrauchs vereinbar machen. Eine nachhaltige Innovationsstrategie für den Energiebereich hat eine international besetzte Forschergruppe der Europäischen Akademie zur Erforschung von Folgen wissenschaftlich-technischer Entwicklungen Bad Neuenahr-Ahrweiler GmbH mit der Studie „Nachhaltige Entwicklung und Innovation im Energiebereich“" ausgearbeitet. Die Studie wurde im Oktober 2002 der Öffentlichkeit vorgestellt.

\section{Grundbegriffe}

\subsection{Energie}

Unter dem Energiesystem (eines Landes oder der Erde insgesamt) versteht man die gesamte Struktur der genutzten Primärenergieressourcen, der Infrastruktur zu deren Verteilung und Umwandlung in Endenergie und der spezifischen Nachfragestruktur der so genannten Energiedienstleistungen. Im Hinblick auf die Wertigkeit der Energie spielt insbesondere die Unterscheidung zwischen Bedarf an Wärme bzw. Arbeit eine Rolle, ferner die Aufteilung zwischen stationärem und mobilem Bedarf und die Rolle der Elektrizität. Angebots- und Bedarfsstruktur bestimmen zusammen das Potenzial zur Veränderung eines gegebenen Energiesystems.

\subsection{Nachhaltige Entwicklung}

Die verschiedenen Konzepte von der ,schwachen“ bis hin zur „sehr starken“ Nachhaltigkeit unterscheiden sich im Hinblick auf Substitutions- und Komplementaritätsannahmen in Bezug auf menschengemachtes Kapital und
Naturkapital. Dieser Studie liegt ein Konzept kritischer Nachhaltigkeit zugrunde, das sich auf der Grundlage des Konzepts des kritischen Naturkapitals auf wenige, aber entscheidende und in diesem Sinne kritische „Leitplanken“ oder „Engpässe“ konzentriert. Dieses Verständnis von Nachhaltigkeit schafft damit eine Verbindung zur weit entwickelten Diskussion der Setzung von Umweltstandards.

\subsection{Innovation}

Innovationen bezeichnen die Durchsetzung neuer Problemlösungen am Markt, verbunden mit neuen Faktorkombinationen. Nachhaltige Innovation bezeichnet Faktorkombinationen und neue Problemlösungen, die zu einer Senkung von Umweltbelastung und Ressourcenverbrauch führen, ohne dass dadurch Einschränkungen bei anderen gesellschaftlichen Zielen erforderlich werden. Dazu gehören nicht nur neue technologische Lösungen (Prozesse, Produkte), sondern auch neue Dienstleistungen und neue Organisationsformen.

\section{Kontext}

Veränderungen des Energiesystems müssen nicht nur einer Zielgröße - etwa der Reduktion von Treibhausgass-Emissionen - genügen, sondern einer ganzen Reihe von Zielen. Ein Zielbündel für einen nachhaltigen Umbau des Energiesystems berücksichtigt Aspekte der Ressourcenverfügbarkeit (z. B. Beschaffungssicherheit) und des Energiesystems (z. B. Verlässlichkeit und Optionsoffenheit) ebenso wie Umweltaspekte (z. B. Emissionen und Flächenverbrauch).

Die Reduzierung der $\mathrm{CO}_{2}$-Emissionen lässt sich als „Leitindikator“ für dieses Zielbündel verwenden, der einer notwendigen Bedingung entspricht und durch weitere Indikatoren (Flächenverbrauch, Optionsoffenheit u. ä.) als hinreichende Bedingungen ergänzt werden muss.

Um nachhaltige Innovation zu verstärken, sind Kenntnisse über Innovationsdeterminanten erforderlich. Ausmaß, Richtung und Geschwindigkeit der Innovationsaktivität in einer Volkswirtschaft hängen von einer Vielzahl von Faktoren ab, die zusammenfassend auch als „Nationales Innovationssystem“ bezeichnet werden und die weit über die Forschungs- und 
Entwicklungspolitik hinaus bis hin zum Steuerund Bildungssystem reichen. Im Zuge der europäischen Integration ist es in bestimmten Bereichen inzwischen sinnvoller, von einem europäischen Innovationssystem zu sprechen. Diesen gesamten Kontext gilt es neu zu gestalten, wenn die Innovationsaktivität auf Umwelt- und Ressourcenschonung ausgerichtet werden soll. Notwendig erscheint hier eine innovationspolitische Doppelstrategie, die einerseits auf kurzfristige Wirkungen abzielt, andererseits aber auch längerfristig wirksame Weichenstellungen vornimmt.

Zur Operationalisierung der Nachhaltigkeitsziele werden zwei Konzepte vorgeschlagen: (1) Die Zeit sicherer Praxis geht davon aus, dass jede gesellschaftliche Tätigkeit daraufhin analysiert werden kann, wie lange sich diese hypothetischerweise unverändert fortsetzen ließe, bis sie (z. B. aus Gründen der Ressourcen-Reichweite, der Umweltbelastung etc.) an ihre eigenen Grenzen stieße. (2) Die Trägheit des Energiesystems lässt sich als jene Zeit definieren, die es benötigt, um ein System signifikant zu verändern. Für den Fall des heutigen, durch den Verbrauch fossiler Brennstoffe dominierten Systems wäre eine solche Veränderung zum Beispiel die Umstellung auf erneuerbare Energieressourcen.

Mit diesen Konzepten lässt sich das Ziel der Nachhaltigkeit folgendermaßen definieren:

- Eine Praxis (z. B. eine Energiepraxis) ist dann nachhaltig, wenn die Zeit sicherer Praxis konstant bleibt oder wächst (Prinzip der konstanten Zeit sicherer Praxis).

- Die Zeit sicherer Praxis muss größer sein als die Trägheit des betrachteten Systems.

Auf die Energie angewendet bedeutet dies, dass sich ein nachhaltiges Energiesystem auf zwei Pfeiler stützen muss: (1) auf den effizienten Umgang mit Energie und (2) auf die vermehrte Nutzung der solaren Ressourcen. Aufgrund der heute bekannten Technologien könnte ein westlicher Lebensstandard mit einem Energieverbrauch von 2.000 Watt pro Person (Durchschnittsdauerleistung) erreicht werden. (Der Verbrauch in den Industrieländern liegt heute zwischen 4.000 und 10.000 Watt pro Person.) Dieser Bedarf könnte weitgehend durch erneuerbare Ressourcen gedeckt werden. Die 2000 Watt-Benchmark bildet die
Grundlage für die Strategie der Studie. Es gibt Anlass für die Annahme, dass genügend Zeit für einen solchen Wandel bleibt, wenn er jetzt energisch in Angriff genommen wird. Das technische Potenzial ist, wie in drei exemplarischen Szenarien durchgeführt, vorhanden.

\section{Strategie}

In dieser Situation ist es die zentrale politische Führungsaufgabe, Energie wieder als strategische Priorität auf die Tagesordnung von Wirtschaft, Bürgern und Politik zu bringen. Diese Aufgabe hat zwei maßgebliche Dimensionen: eine inhaltliche und eine prozedurale. Die erste Dimension wird durch die Frage bestimmt: Was ist zu tun?, die zweite durch die Frage: Wie setze ich es durch, wie überwinde ich die einschlägigen Zielkonflikte?

Eine Strategie muss die Potenziale im Hinblick auf die 2000-Watt-Benchmark aktivieren, ohne an konfligierenden Zielen $\mathrm{zu}$ scheitern. Aus der in der Studie vorgenommenen Analyse lässt sich eine solche Strategie entwickeln, die durch ein Bündel von Maßnahmen zur Förderung nachhaltiger Energieinnovationen geeignet ist:

1. Kern dieser Strategie ist die Beschleunigung nachhaltiger Energieinnovationen durch maßgeschneiderte Fördermaßnahmen für unterschiedliche Phasen ihres Lebenszyklus in Bezug auf ein Lernkurvenmodell. Zu Beginn des Lebenszyklus sollen Subventionen dazu beitragen, dass die Kostenvorteile der Skaleneffekte überhaupt erreicht werden können, indem Unternehmen sich rascher auf der „Lernkurve“ von Kostensenkungen bewegen. In einer späteren Phase sollen Selbstverpflichtungen als Verhandlungslösungen oder einseitige Selbstverpflichtungen der Industrie zu beschleunigter Marktdurchdringung führen. Es geht hier vornehmlich um energieeffiziente Technologien, die in der Phase der Markteinführung sind, d. h. es existieren Pilotprojekte und Demonstrationsvorhaben, aber es ist jetzt notwendig, die „early adapter" zu gewinnen und industrielle Produktions- und Servicestrukturen durch größere Stückzahlen zu entwickeln. Diese Subventionen sind Anschubfinanzierungen. Es lässt sich nämlich zeigen, dass in der Mehrzahl der Fälle die Kosten der saubere 
ren Technik langfristig nicht über denen der alten Technik liegen.

2. Konkret wird für diesen Subventionsansatz auf das niederländische Modell der „Energieliste" zurückgegriffen. Durch eine jährlich aktualisierte Liste förderungswürdiger Techniken wird verhindert, dass eine Subventionierung länger als notwendig Bestand hat. Die in den Niederlanden gemachten Erfahrungen zur Überwindung von Informationsasymmetrien, zur Minimierung von Mitnahmeeffekten und zur ausschließlichen Konzentration auf die wirklich innovativen Techniken müssen berücksichtigt werden.

3. Die Nachfrage sollte zusätzlich über staatliche Beschaffungsprogramme stimuliert werden. So könnte im Zuge der üblichen Neubau-, Modernisierungs- und Reparaturmaßnahmen innerhalb der nächsten 5-7 Jahre auf jedem dritten staatlichen Gebäude eine Photovoltaik-Anlage installiert werden.

4. Notwendig ist eine Ausweitung der Grundlagenforschung im Bereich der Energietechnologien, um einen ständigen Strom neuen Wissens - angefangen von der Kernfusion bis hin zur Solarenergie - zu gewährleisten. Diese Aufgabe fällt eindeutig in den Zuständigkeitsbereich der Regierung. Eine Reduktion der entsprechenden Förderung (vgl. 6. Rahmenprogramm für Forschung, technologische Entwicklung und Demonstration (2002-2006) der EU) ist eindeutig der falsche Weg.

5. Darüber hinaus sollte sich die Regierung im Bereich geeigneter Bildung und infrastruktureller Kompetenzbildung für neue Energietechniken engagieren.

6. In den Haushalten und im Bereich der Mobilität steigt der Gesamtverbrauch weiter an. Es stehen einige Ansätze zur Verfügung, mit denen sich die derzeitige Nachlässigkeit der Konsumenten beim Energieverbrauch beeinflussen ließe. Die Instrumente zur Regulierung der Haushalte als Hauptverursacher von Energieverbrauch, Emissionen und Abfällen sind wesentlich weniger gut entwikkelt als im Industriebereich, z. B. die IVURichtlinie (Integrierte Vermeidung und Verminderung der Umweltverschmutzung/IPPC Directive) mit der Verpflichtung zur Nutzung der ,besten verfügbaren Techniken". Verbraucher sind nicht nur durch das Trittbrettfahrer-Dilemma eingeschränkt, sondern auch durch Informationsmängel, die sie daran hindern, fundierte Entscheidungen $\mathrm{zu}$ treffen. Hier kann eine wirksame und glaubwürdige Kennzeichnung (Labeling) einschließlich eines „Greenpricing“ von Strom aus regenerativen Quellen Abhilfe schaffen. Der geringe Erfolg bisheriger Ansätze lag in der Überflutung des Marktes mit Kennzeichnungen, so dass es selbst staatlichen Kennzeichnungen nicht wirklich gelang, den Unterschied zwischen nachhaltigen und nicht nachhaltigen Produkten deutlich zu machen. In einigen Fällen ist dies auf Lobbyarbeit der Industrie zurückzuführen, in anderen auf einen Mangel an brauchbaren Unterscheidungskriterien.

7. Es könnte viel versprechender sein, zur Beschleunigung der Diffusion von Energieinnovationen in Haushalten auf ,gesellschaftliche und organisatorische Innovati-

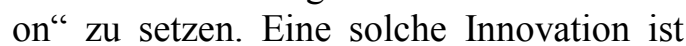
das Experiment von Versorgungsunternehmen - häufig in einer Partnerschaft zwischen öffentlichen Institutionen und Unternehmen -, sich als Dienstleister zu positionieren. Ein solcher Schritt befreit sie von dem Zwang, immer mehr Energie verkaufen zu müssen. Stattdessen können sie profitable Dienstleistungen für einen effizienten Energieverbrauch anbieten.

8. Im Verkehrsbereich müssen die im Vergleich zu Pkw und Lkw energieeffizienteren Verkehrsträger komfortablere und schnellere Logistik- oder Mobilitätsketten aufbauen, um wettbewerbsfähig sein zu können. Höhere Marktanteile lassen sich nicht mit Verbesserungen der einzelnen Komponenten, sondern nur mit einer Überarbeitung des gesamten Transportprozesses erreichen. Dies erfordert innovative Pakete, beispielsweise die Verknüpfung des Eisenbahnverkehrs mit Car-Sharing und Informationsdiensten.

9. Aber auch hier darf man nicht ausschließlich auf die Technologieentwicklung schauen: Mindestens genauso wichtig ist die Kompatibilität mit bestehenden Infrastrukturen und Prozessen sowie die Integration in das Stromnetz, sowohl auf der lokalen (um diskontinuierliche Ressourcen wie Wind zu kompensieren) als auch auf der europäischen Ebene (beispielsweise im 
Winter mit Wasserkraft gewonnene Energie aus Skandinavien in den Süden und im Sommer photovoltaisch gewonnene Energie aus Italien in den Norden).

10. Dem Thema Energie muss auf allen politischen Ebenen wieder höchste Priorität eingeräumt werden.

11. Neue Institutionen werden skeptisch beurteilt. Im Gegensatz zu spezialisierten Behörden, angefangen von der Zentralbank bis zu einer Kartellbehörde, betrifft die Nachhaltigkeit jeden Lebensaspekt und kann nicht vom Kern demokratischer Politik getrennt werden. Es reicht im Allgemeinen, bestehende Institutionen zu vernetzen, um das Konzept nachhaltiger Entwicklung in ihre speziellen Aufgabenbereiche zu integrieren. Deshalb schlagen wir die Bildung einer ,Allianz für nachhaltige Energieinnovationen" vor.

12. Nachhaltige Energieinnovationen sind Voraussetzung für die Entwicklung von Energiesystemen in Entwicklungs- und Schwellenländern. Die wichtigsten Besonderheiten betreffen den Mangel an Kompetenz und Infrastruktur, das begrenzte kommerzielle Energieangebot und den ineffizienten Verbrauch, insbesondere von Holz. Viele moderne Techniken der Energiegewinnung aus regenerativen Quellen, insbesondere aus Windkraft, Biomasse und Sonnenenergie, sollten in diesen Ländern eingesetzt werden. Bevor sie jedoch in der Praxis von Belang sein können, müssen Kompetenzen und Infrastruktur aufgebaut werden. Im dünn besiedelten ländlichen Raum außerhalb der Städte sind die dezentralen Technologien in vielen Fällen wesentlich sinnvoller als zentrale Versorgungen. Aber auch hier sind umfangreiche und gezielte Maßnahmen notwendig.

Die im Oktober 2002 der Öffentlichkeit präsentierte Studie ist im Buchhandel erhältlich (eine Zusammenfassung steht als pdf-Datei auf den Webseiten der Akademie zum download bereit):

U. Steger, W. Achterberg, K. Blok, H. Bode, W. Frenz, C.Gather, G. Hanekamp, D. Imboden, M. Jahnke, M. Kost, R. Kurz, H.G. Nutzinger, Th. Ziesemer: Nachhaltige Entwicklung und Innovation im Energiebereich. Berlin: Springer, 2002.

\section{Kontakt}

Dr. Gerd Hanekamp

Europäische Akademie zur Erforschung von Folgen wissenschaftlich-technischer Entwicklungen

Bad Neuenahr-Ahrweiler GmbH

Wilhelmstr. 56, 53474 Bad Neuenahr-Ahrweiler

E-Mail: Gerd.Hanekamp@dlr.de

Internet: http://www.europaeische-akademie-aw.de

)

\section{Wandel der Arbeits- und Le- bensbedingungen im Multime- dia-Bereich aus der Gender- perspektive}

\author{
von Bettina-Johanna Krings, ITAS
}

Das Projekt „Wandel der Arbeits- und Lebensbedingungen im Multimedia-Bereich aus der Genderperspektive" wurde im Rahmen des Forschungsbereiches „Neue Technologien und Informationsgesellschaft" des ITAS durchgeführt und befasste sich mit der spezifischen Fragestellung, welche Auswirkungen die Informatisierung der Arbeitsstrukturen auf die Lebensführung der Beschäftigten hat. Diese Fragestellung wurde aus der Perspektive der Genderforschung bearbeitet. Die Studie wurde im Auftrag des Sozialministeriums Baden-Württemberg durchgeführt. Das methodische Kernstück der Studie ist die Verknüpfung der theoretischen Diskussion über die Re-Organisation der Arbeit mit Fallstudien zu den subjektiven Arbeits- und Lebenserfahrungen von Frauen und Männern in der Multimediabranche.

Die Ergebnisse dieser Fallstudien zeigen, wie notwendig eine geschlechtsspezifische Differenzierung in dieser Fragestellung ist, da sich der vollziehende gesellschaftliche Wandel derzeit eher ,hinter verschlossenen Türen" abspielt und in der Öffentlichkeit, also in der Erwerbsarbeit, wenig sichtbar ist.

\section{Fragestellung des Projekts}

Schon im Rahmen der arbeitssoziologischen Diskussion der 80er Jahre galten die Potenziale der Informations- und Kommunikationstechnologien (IuK) als richtungsweisend für die Modernisierung der Arbeitsstrukturen. Als zentrale Kategorie, die diese Prozesse beschreiben sollte, wurde hier der Begriff der 